\title{
SMALL SETS OF INFINITE TYPE ARE BENIGN FOR THE $\bar{\partial}$-NEUMANN PROBLEM
}

\author{
HAROLD P. BOAS
}

(Communicated by Irwin Kra)

\begin{abstract}
An explicit construction shows that the $\bar{\partial}$-Neumann operator and the Bergman and Szegö projections are globally regular in every smooth bounded pseudoconvex domain whose set of boundary points of infinite type has Hausdorff two-dimensional measure equal to zero. On the other hand there are examples of domains with globally regular $\bar{\partial}$-Neumann operator but whose infinite-type points fill out an open subset of the boundary.
\end{abstract}

Is the $\bar{\partial}$-Neumann problem globally regular on every smooth bounded pseudoconvex domain in $\mathbf{C}^{n}$ ? Although this basic question remains open, some sufficient geometric conditions on the domain for an affirmative answer are known. For instance the answer is yes if the boundary of the domain repels analytic discs in a suitable differential-geometric sense.

Here I show that the answer is yes if the boundary of the domain excludes analytic discs in a suitable measure-theoretic sense. This result was recently obtained by Sibony [25] as a corollary of a general theory. What is new in this paper is the simple proof, an elementary and explicit construction.

It was a quarter of a century ago that Kohn $[\mathbf{1 7}]$ made the first breakthrough on the $\bar{\partial}$-Neumann problem by establishing both global and local regularity in case the domain is strongly pseudoconvex. More recently Catlin $[8]$ proved that a subelliptic estimate holds near a boundary point $p$ if and only if $p$ is a point of finite type in the sense of D'Angelo [11], meaning that complex varieties have bounded order of contact with the boundary at $p$. Consequently the $\bar{\partial}$-Neumann problem is locally regular near points of finite type, and so if obstructions to global regularity exist then they must lie in the set of boundary points of infinite type.

Catlin [7] also found a more general condition, property $(\mathrm{P})$, guaranteeing global regularity of the $\bar{\partial}$-Neumann problem. This property says that there exist bounded plurisubharmonic functions having all eigenvalues of their complex Hessian arbitrarily large at the boundary. Catlin proved that property $(\mathrm{P})$ is satisfied by domains that are regular in the sense of Diederich and Fornæss $[13,15]$. For instance a domain whose weakly pseudoconvex boundary points lie in a totally real submanifold is regular, but a domain that contains a complex disc in its boundary is not.

Subsequently Sibony [25] introduced the notion of $B$-regularity. The boundary of a smooth bounded pseudoconvex domain is $B$-regular if every continuous function on the boundary is the boundary value of a function plurisubharmonic

Received by the editors September 8, 1987.

1980 Mathematics Subject Classification (1985 Revision). Primary 32F 20.

Key words and phrases. $\bar{\partial}$-Neumann problem, Bergman projection, global regularity.

Partially supported by NSF grant DMS-8701038. 
on the interior of the domain. By studying Choquet theory for plurisubharmonic functions, Sibony showed that this property is equivalent to Catlin's property (P). This enabled him to give some new examples of domains with globally regular $\bar{\partial}$-Neumann problem. One corollary of Sibony's elegant abstract theory is the following result (stated in [25, Remarque, p. 310] in different language): if the set of points of infinite type in the boundary of a smooth bounded pseudoconvex domain has Hausdorff two-dimensional measure equal to zero, then the $\bar{\partial}$-Neumann problem is globally regular.

To prove this assertion, I explicitly construct a bounded plurisubharmonic function with large Hessian near the set of points of infinite type. I also localize the statement: even if the set of points of infinite type has large Hausdorff dimension, the $\bar{\partial}$-Neumann operator is regular in a neighborhood of any separated piece of this set having Hausdorff two-dimensional measure equal to zero.

Using a different method, I give some simple examples of domains that have analytic discs in the boundary (and hence do not have $B$-regular boundary), but nonetheless have globally regular $\bar{\partial}$-Neumann problem. To do this I use the equivalence in $\mathbf{C}^{2}$ of global regularity of the $\bar{\partial}$-Neumann operator and of the Bergman projection.

The connection with the Bergman projection is an important application of the $\bar{\partial}$-Neumann theory, for it leads to results on the boundary behavior of holomorphic mappings. Global regularity of the $\bar{\partial}$-Neumann problem implies global regularity of the Bergman projection, which is condition $R$ of Bell and Ligocka. A biholomorphic mapping between two smooth bounded domains satisfying condition $R$ extends smoothly to the boundaries [4]. For elaboration of this theme see $[\mathbf{3}, \mathbf{1 4}]$, and their references.

1. Local property (P). Suppose $\Omega$ is a smooth bounded pseudoconvex domain in $\mathbf{C}^{n}$. Let $\bar{\partial}^{*}$ be the $L^{2}$ adjoint of $\bar{\partial}$. The $\bar{\partial}$-Neumann operator $N$ is the bounded operator on the space of $(0,1)$-forms with $L^{2}$ coefficients that is the inverse of the closed, densely defined, unbounded operator $\bar{\partial} \bar{\partial}^{*}+\bar{\partial}^{*} \bar{\partial}$ (the complex Laplacian). One says that the $\bar{\partial}$-Neumann problem is globally regular if $N f$ has coefficients in $C^{\infty}(\bar{\Omega})$ whenever $f$ does. This of course implies that when $f$ is a $\bar{\partial}$-closed $(0,1)$-form with coefficients in $C^{\infty}(\bar{\Omega})$, then the canonical solution orthogonal to holomorphic functions of the equation $\bar{\partial} u=f$ also lies in $C^{\infty}(\bar{\Omega})$; indeed this solution is $u=\vartheta N f$, where $\vartheta$ is the formal adjoint of $\bar{\partial}$. For more details on the $\bar{\partial}$-Neumann problem see [16].

The following assertion is essentially a localized version of Catlin's result [7] that property $(\mathrm{P})$ implies global regularity.

Proposition. Let $\Omega \subset \mathbf{C}^{n}$ be a smooth bounded pseudoconvex domain. Let $S$ be a closed subset of the boundary $b \Omega$ having an open neighborhood $V$ in $\mathbf{C}^{n}$ such that all points in $V \cap b \Omega \backslash S$ are of finite type. Suppose that for every positive $M$ there exist an open neighborhood $U$ of $S$ in $\mathbf{C}^{n}$ and a function $\lambda$ in $C^{2}(U)$ such that $0 \leq \lambda \leq 1$ and

$$
\sum_{j, k=1}^{n} \frac{\partial^{2} \lambda(z)}{\partial z_{j} \partial \bar{z}_{k}} \xi_{j} \bar{\xi}_{k} \geq M|\xi|^{2}
$$

for all $z$ in $U$ and all $\xi$ in $\mathbf{C}^{n}$. If the $(0,1)$-form $f$ has coefficients smooth in a neighborhood of $S$ in $\bar{\Omega}$, then so does $N f$. 
ProOF. Multiplying $\lambda$ by a suitable cut-off function and shrinking $U$ slightly, we may assume that $\lambda$ is defined, twice continuously differentiable, and bounded between 0 and 1 in a neighborhood of $\bar{\Omega}$. For such $\lambda$ a fundamental inequality of Catlin [7] says that

$$
\int_{\Omega} \sum_{j, k=1}^{n} \frac{\partial^{2} \lambda}{\partial z_{j} \partial \bar{z}_{k}} g_{j} \bar{g}_{k} \leq 16\left(\|\bar{\partial} g\|^{2}+\|\vartheta g\|^{2}\right)
$$

if $g$ is a $(0,1)$-form in the common domain of $\bar{\partial}$ and $\bar{\partial}^{*}$. Here $\|\cdot\|$ denotes the norm in $L^{2}(\Omega)$. The hypothesis of the Proposition implies that

$$
M\|g\|^{2} \leq 16\left(\|\bar{\partial} g\|^{2}+\|\vartheta g\|^{2}\right)
$$

if $g$ is supported in the neighborhood $U$ corresponding to $M$.

We may assume without loss of generality that $f$ is smooth on $\bar{V}$ and that $U \subset V$. Let $\phi$ and $\psi$ be cutoff functions in $C_{0}^{\infty}\left(\mathbf{C}^{n}\right)$ such that $\phi$ is supported in $U$ and is identically equal to one in a neighborhood of $S$, while $\psi$ is supported in $U \backslash S$ and is identically equal to one on the support of $\nabla \phi$. Take $g$ in (1) to be equal to $\phi$ times a (tangential) derivative of $N f$ of order $s$. Applying the machinery of Kohn and Nirenberg [20], one finds

$$
M\|\phi N f\|_{s}^{2} \leq K\left(\|\phi f\|_{s}^{2}+\|\phi N f\|_{s}^{2}\right)+C\left(\|\psi N f\|_{s}^{2}+\|\psi f\|_{s}^{2}\right),
$$

where the first constant $K$ is independent of $\phi$, and $\|\cdot\|_{s}$ denotes the norm in the Sobolev space $W^{s}(\Omega)$. (Roughly speaking the machine runs like this: commuting derivatives and integrating by parts on the right-hand side of (1) makes $(\vartheta \bar{\partial}+\bar{\partial} \vartheta) N f=f$ appear, which gives a term of the form

$$
\left|\left\langle D^{s} \phi f, D^{s} \phi N f\right\rangle\right| \leq K\left(\|\phi f\|_{s}^{2}+\|\phi N f\|_{s}^{2}\right) .
$$

Commuting $\vartheta$ or $\bar{\partial}$ with a derivative of order $s$ makes an error term of the form $\|\phi N f\|_{s}^{2}$, and commuting any derivative with $\phi$ makes an error that lives on the support of $\psi$. The normal derivative is controlled by $\bar{\partial}, \vartheta$, and tangential derivatives.)

Since $b \Omega \cap \operatorname{supp} \psi$ is contained in the set of points of finite type, where the $\bar{\partial}$ Neumann problem is locally regular by Catlin's theorem, the second term on the right-hand side is dominated by the sum of the squares of the $W^{s}(U \cap \Omega)$ norm of $f$ and the $L^{2}(\Omega)$ norm of $f$. If $N f$ is known a priori to be smooth in $U \cap \bar{\Omega}$, then the term $\|\phi N f\|_{s}^{2}$ on the right-hand side can be absorbed into the left-hand side as soon as $M>K$. This gives the estimate

$$
\|\phi N f\|_{s} \leq C\left(\|f\|_{s, U \cap \Omega}+\|f\|\right) .
$$

Let $W \subset \subset V$ be a fixed open neighborhood of $S$, independent of $\phi$. The previous estimate combined with the local regularity of $N$ on the set $W \cap \operatorname{supp}(1-\phi)$ gives

$$
\|N f\|_{s, W \cap \Omega} \leq C\left(\|f\|_{s, V \cap \Omega}+\|f\|\right)
$$

To finish the proof it remains to remove the hypothesis that $N f$ is a priori smooth. This can be done by standard techniques: either by the method of elliptic regularization [20], or (in this case) by approximating $\Omega$ from inside by strongly pseudoconvex domains (cf. [5]). From the last estimate, which holds for every positive integer $s$, it follows by Sobolev's lemma that $N f \in C^{\infty}(W \cap \bar{\Omega})$. 


\section{The main result.}

THEOREM. Let $\Omega$ be a smooth bounded pseudoconvex domain in $\mathbf{C}^{n}$. Suppose that the set of boundary points of infinite type is the union of two disjoint closed sets $S$ and $T$, and that $S$ has Hausdorff two-dimensional measure equal to zero. If the $(0,1)$-form $f$ has coefficients smooth in a neighborhood of $S$ in $\bar{\Omega}$, then so does $N f$.

COROLLARY (AFTER SIBONY [25]). If the set of points of infinite type in the boundary of a smooth bounded pseudoconvex domain has Hausdorff two-dimensional measure equal to zero, then the $\bar{\partial}-$ Neumann problem is globally regular, and so are the Bergman and Szegö projections.

PrOOF. The first conclusion of the Corollary follows by taking $T=\varnothing$ and using the local regularity at the points of finite type. (Note that the set of points of infinite type is closed [11].) Global regularity of the Bergman projection follows immediately from global regularity of the $\bar{\partial}$-Neumann operator (see the proof of the Lemma below). I will show in the proof of the Theorem that local property (P) holds in a neighborhood of $S$ in the sense of the Proposition. Since the rest of the boundary consists of points of finite type, it follows $[\mathbf{8}, \mathbf{2 5}]$ that global property $(\mathrm{P})$ holds, and hence [5, Theorem 4.1] the Szegö projection is globally regular.

The Theorem admits a very simple proof in case $S$ has Hausdorff $\alpha$-dimensional measure equal to zero for some $\alpha$ strictly less than two. Indeed if $D$ is the diameter of $\Omega$ and $M$ is a fixed positive number, then this hypothesis implies that there is a finite set of points $p_{m}$ in $S$ and corresponding radii $r_{m}$ such that

(1) the open balls of center $p_{m}$ and radius $r_{m}$ cover the set $S$;

(2) $\sum r_{m}^{\alpha}<1$; and

(3) if $r=\max r_{m}$ then this maximum radius $r$ is small: namely, $4 r^{2-\alpha} \log \left(1+4 D^{2} / r^{2}\right)<1 / M$ and $r<D \exp (\alpha-2)^{-1}$, which entails $4 r_{m}^{2-\alpha} \log \left(1+4 D^{2} / r_{m}^{2}\right)<1 / M$ for all $m$.

Let $U$ be the union of the balls with center $p_{m}$ and radius $r_{m}$, and set

$$
\lambda(z)=\sum_{m} \frac{r_{m}^{\alpha} \log \left(1+\left|z-p_{m}\right|^{2} / r_{m}^{2}\right)}{\log \left(1+4 D^{2} / r_{m}^{2}\right)} .
$$

By construction $\lambda$ is a smooth, strictly plurisubharmonic function in $\mathbf{C}^{n}$, and $0 \leq$ $\lambda(z) \leq 1$ when $z \in U$. Moreover if $z \in U$, then $\left|z-p_{m}\right|<r_{m}$ for some $m$, and so

$$
\sum_{j, k=1}^{n} \frac{\partial^{2} \lambda(z)}{\partial z_{j} \partial \bar{z}_{k}} \xi_{j} \bar{\xi}_{k} \geq \frac{|\xi|^{2}}{4 r_{m}^{2-\alpha} \log \left(1+4 D^{2} / r_{m}^{2}\right)} \geq M|\xi|^{2}
$$

for all $\xi$ in $\mathbf{C}^{n}$. Since by assumption $V:=\mathbf{C}^{n} \backslash T$ is a neighborhood of $S$ containing no other points of infinite type, the hypotheses of the Proposition are satisfied, and so the conclusion of the Theorem follows.

In the general case a slightly more elaborate construction is required. To begin we may reduce to the case of one variable. Indeed if $S_{j}$ denotes the projection of $S$ onto the $z_{j}$ coordinate plane, then $S_{j}$ still has Hausdorff two-dimensional measure zero; and if there exists a smooth subharmonic function $\lambda_{j}$ in a neighborhood of $S_{j}$ (in C) such that $0 \leq \lambda_{j} \leq 1$ and $\Delta \lambda_{j} \geq M$, then $\lambda\left(z_{1}, \ldots, z_{n}\right):=(1 / n) \sum_{j=1}^{n} \lambda_{j}\left(z_{j}\right)$ is a 
smooth plurisubharmonic function in a neighborhood of $S$ satisfying the hypothesis of the Proposition (with $M$ replaced by $M / 4 n$ ).

Thus we may assume that $S$ is a subset of the complex plane $\mathbf{C}$ of planar Lebesgue measure zero, and we are to construct a smooth subharmonic function $\lambda$ in a neighborhood of $S$ such that $0 \leq \lambda \leq 1$ and $\Delta \lambda \geq M$. There is a finite set of points $p_{m}$ in $S$ and corresponding radii $r_{m}$ such that

(1) the discs $B\left(p_{m}, r_{m}\right)$ of center $p_{m}$ and radius $r_{m}$ cover $S$; and

(2) $\sum r_{m}^{2}<1 / 2 M$.

It will suffice to construct a smooth subharmonic function $\phi_{m}$ defined in an open set $U_{m}$ containing $S \cup B\left(p_{m}, r_{m}\right)$ such that $0 \leq \phi_{m} \leq 1$ in $U_{m}$ and $\Delta \phi_{m} \geq$ $1 / 2 r_{m}^{2}$ in $B\left(p_{m}, r_{m}\right)$. For then $\lambda:=2 M \sum r_{m}^{2} \phi_{m}$ will have the required properties in $\bigcap_{m} U_{m}$ if we assume, as we may, that each $U_{k} \subset \bigcup_{m} B\left(p_{m}, r_{m}\right)$.

So fix a point $p$ in $S$ and a radius $r$. I wish to construct, in a reasonably explicit way, an open set $U$ containing $S \cup B(p, r)$ and a smooth subharmonic function $\phi$ on $U$ with $0 \leq \phi \leq 1$ and with $\Delta \phi \geq 1 / 2 r^{2}$ on $B(p, r)$. Let $\left\{U_{j}\right\}$ be a decreasing sequence of open sets, each bounded by a finite number of closed analytic curves, whose intersection is $S \cup \overline{B(p, 2 r)}$; and let $V_{j}$ be the connected component of $U_{j}$ containing $p$. I will construct the required $\phi$ from the solutions on $V_{j}$ of two extremal area problems.

I follow [22, Chapter VII, §4]. If $D$ is a domain containing $p$ then the inner area of $D$ is the minimum of $\int_{D}\left|g^{\prime}(z)\right|^{2} d x d y$ over the class of analytic functions $g$ in $D$ normalized by $g^{\prime}(p)=1$ and $g(p)=p$; the unique extremal function achieving the minimum is denoted $M^{*}(z)$. It is easy to see that when $D$ is a disc of radius $R$ centered at $p$, the extremal function $M^{*}$ is the identity and the inner area equals the usual area $\pi R^{2}$. The outer area of $D$ is the maximum, taken over the class of univalent analytic mappings from $D$ into the sphere whose difference from $1 /(z-p)$ vanishes at $p$, of the area of the complement of the image of $D$; the unique extremal function achieving the maximum is denoted $N(z)$. It is easy to see that when $D$ is a disc of radius $R$ centered at $p$, the extremal function $N(z)$ is inversion with respect to $p$ and the outer area is $\pi / R^{2}$. When $D$ is bounded by a finite number of closed analytic curves, then the analytic function $F(z):=(\pi / A) M^{* \prime}(z) / N^{\prime}(z)$, which has a double zero at $p(A$ being the inner area of $D)$, turns out to map $D$ onto the unit disc $[\mathbf{2 2}$, p. 340, ex. 4] with multiplicity twice the connectivity of $D$.

I claim that the functions $F_{j}(z)$ associated in this way to the domains $V_{j}$ converge uniformly on compact subsets of the disc $B(p, 2 r)$ to the corresponding function $-(z-p)^{2} / 4 r^{2}$ for this disc. Granted this claim, we may take for the required subharmonic function $\phi$ simply $\left|F_{j}\right|$ on $V_{j}$ and zero on the other components of $U_{j}$ for a sufficiently large $j$, since $\Delta\left|F_{j}\right|$ converges uniformly on $B(p, r)$ to $1 / r^{2}$.

To complete the proof, it suffices to show that the inner area of $V_{j}$ converges to the (inner) area of $B(p, 2 r)$ and that the extremal functions $M_{j}^{*}(z)$ and $N_{j}(z)$ converge normally in $B(p, 2 r)$ to the corresponding extremal functions for this disc. By its definition the inner area of $V_{j}$ is trapped between the usual Lebesgue area of $V_{j}$ and the (inner) area of $B(p, 2 r)$. But since $S$ has measure zero, the Lebesgue area of $V_{j}$ converges to the (Lebesgue = inner) area of $B(p, 2 r)$; so the inner area of $V_{j}$ converges as well to the same limit. The extremal functions $M_{j}^{*}$ form a normal family on $B(p, 2 r)$. If $g$ is the limit of a subsequence, then by Fatou's lemma the integral of $\left|g^{\prime}\right|^{2}$ over $B(p, 2 r)$ does not exceed the limit as $j \rightarrow \infty$ of the inner area 
of $V_{j}$, which was just shown to equal the (inner) area of $B(p, 2 r)$. Therefore $g$ must equal the unique extremal function on the domain $B(p, 2 r)$, namely the identity. Since the normal family $\left\{M_{j}^{*}\right\}$ has a single limit point, the whole sequence converges to the identity. The family $\left\{N_{j}\right\}$ is normal because the $N_{j}$ are univalent; let $h$ be the limit of a subsequence. The integral

$$
\iint_{V_{j}}\left|N_{j}^{\prime}(z)+\frac{1}{(z-p)^{2}}\right|^{2} d x d y
$$

equals $\left[\mathbf{2 2}\right.$, p. 363] the outer area of $V_{j}$ minus the area of the complement of the image of $V_{j}$ under inversion with respect to $p$. By monotone convergence and the definition of the outer area, this difference has a nonpositive limit as $j \rightarrow \infty$, so by Fatou's lemma

$$
\iint_{E(p, 2 r)}\left|h^{\prime}(z)+\frac{1}{(z-p)^{2}}\right|^{2} d x d y=0 .
$$

Accordingly the family $\left\{N_{j}\right\}$ has a unique limit point $h$, which is inversion with respect to $p$. This completes the proof.

\section{Remarks and examples.}

EXAMPLE 1. Let $F$ be the Cantor set constructed not by removing from the unit interval the usual middle thirds, but instead by removing at the $k$ th step from the middle of each of the $2^{k-1}$ remaining intervals $I$ an interval of length $(k+2) / 2(k+1)$ times the length of $I$ (that is, slightly more than half). One readily computes that the set $E:=F \times F$ has Hausdorff dimension one and Hausdorff one-dimensional measure equal to zero. Let $g$ be the Green function with pole at infinity for the complement of $E$ in the Riemann sphere. (This Green function exists since points of Cantor sets are regular for the Dirichlet problem [27, pp. 106-109].) Let $\chi$ be a smooth nonnegative function on the real line that is strongly convex for $x>0$ and tends to zero so rapidly as $x \rightarrow 0^{+}$that $\chi \circ g$ is smooth and vanishes to infinite order on $E$. By Sard's theorem the equation $\chi(g(z))+|w|^{2}<c$ defines a smooth bounded domain $\Omega$ in $\mathbf{C}^{2}$ for almost every positive value of $c$. The Levi form acting on the complex tangent vector $\bar{w}(\partial / \partial z)-\chi^{\prime}(g(z))(\partial g / \partial z)(\partial / \partial w)$ equals

$$
\begin{cases}\left|\frac{\partial g}{\partial z}\right|^{2}\left(|w|^{2} \chi^{\prime \prime}(g(z))+\chi^{\prime}(g(z))^{2}\right), & \text { if } z \notin E ; \\ 0, & \text { if } z \in E ;\end{cases}
$$

and so $\Omega$ is pseudoconvex. Moreover the weakly pseudoconvex boundary points are precisely the ones for which either $z \in E$ (these are points of infinite type) or $z$ is a critical point of $g$ (these are points of finite type). The set of boundary points of infinite type, being the Cartesian product of $E$ with a circle, is a set of Hausdorff dimension two and Hausdorff two-dimensional measure equal to zero. The domain $\Omega$ is not regular in the sense of Diederich, Fornæss, and Catlin because it contains "too much" of a complex disc, in the sense that any smooth submanifold of the boundary that locally contains $E \times\{\sqrt{c}\}$ must have $\partial / \partial z$ as a tangent vector at points of this set. However $\Omega$ does satisfy the hypothesis of the Corollary, and so is globally regular for the $\bar{\partial}$-Neumann problem.

Sibony [25] has given a more sophisticated example of a smooth bounded pseudoconvex domain in $\mathbf{C}^{2}$ that is globally regular for the $\bar{\partial}$-Neumann problem and 
whose weakly pseudoconvex boundary points form a set of positive Hausdorff threedimensional measure. (Since it has property $(\mathrm{P})$, this domain has no complex disc in the boundary.) I give here two examples of domains with globally regular $\bar{\partial}$ Neumann problem but with maximally degenerate boundary: not only does the boundary contain analytic discs, but the points of infinite type even fill out an open subset of the boundary. These examples are based on the following unpublished observation of Catlin, which has been circulating underground for a number of years. With his permission I supply here a proof (my own).

LEMMA (CATLIN). Let $\Omega$ be a smooth bounded pseudoconvex domain in $\mathbf{C}^{2}$. Then the Bergman projection is globally regular if and only if the $\bar{\partial}-$ Neumann operator on $(0,1)$-forms is globally regular.

PROOF. That global regularity of the $\bar{\partial}$-Neumann operator $N$ on $(0,1)$-forms implies global regularity of the Bergman projection $P$ (the orthogonal projection from $L^{2}(\Omega)$ onto the subspace of holomorphic functions) is well known and follows immediately from the formula $P=I-\vartheta N \bar{\partial}$. To prove the converse, the nontrivial part of the Lemma, it evidently suffices to write $N$ as the composition of $(I-P)$ and operators that preserve smoothness up to the boundary. In hopes of clarity I will write on each operator a subscript 0,1 , or 2 according as the operator acts on functions, on $(0,1)$-forms, or on $(0,2)$-forms.

Here is a formula relating the $\bar{\partial}$-Neumann operator $N_{1}$ on $(0,1)$-forms to the Bergman projection $P_{0}$ on functions:

$$
N_{1}=P_{1} \chi_{1}^{(t)} N_{1}^{(t)} \bar{\partial}_{0} \chi_{0}^{(-t)}\left(I_{0}-P_{0}\right) \vartheta_{1}^{(t)} N_{1}^{(t)} P_{1}+\vartheta_{2} N_{2} N_{2} \bar{\partial}_{1}
$$

where $P_{1}=I_{1}-\vartheta_{2} N_{2} \bar{\partial}_{1}$ is the orthogonal projection onto the space of $\bar{\partial}$-closed $(0,1)$-forms $\left(N_{2}\right.$ being the $\bar{\partial}$-Neumann operator at the level of $(0,2)$-forms $), N_{1}^{(t)}$ and $\vartheta_{1}^{(t)}$ denote the $\bar{\partial}$-Neumann operator and the formal adjoint of $\bar{\partial}$ computed for the space of forms with coefficients square-integrable with respect to the weight $\exp \left(-t|\cdot|^{2}\right)$, and $\chi^{(t)}$ is the operator of multiplication by the function $\exp \left(-t|\cdot|^{2}\right)$. To derive (2), start with the formula

$$
N_{1}=\left(\vartheta_{1} N_{1}\right)^{*}\left(\vartheta_{1} N_{1}\right)+\left(\vartheta_{2} N_{2}\right)\left(\vartheta_{2} N_{2}\right)^{*}
$$

due to Range [24]. Obviously the second terms in (2) and (3) agree. Taking the canonical solution in the weighted metric of the equation $\bar{\partial}_{0} u=P_{1} f$ shows that $P_{1}=\bar{\partial}_{0} \vartheta_{1}^{(t)} N_{1}^{(t)} P_{1}$. Applying the operator $\vartheta_{1} N_{1}$ to this equation gives $\vartheta_{1} N_{1}=$ $\left(I_{0}-P_{0}\right) \vartheta_{1}^{(t)} N_{1}^{(t)} P_{1}$, since $\vartheta_{1} N_{1}$ annihilates $\vartheta_{2}$-exact forms. Inserting this in (3) gives (2) since $\left(\vartheta_{1}^{(t)} N_{1}^{(t)}\right)^{*}=\chi_{1}^{(t)} N_{1}^{(t)} \bar{\partial}_{0} \chi_{0}^{(-t)}$ and $\left(I_{0}-P_{0}\right)$ and $P_{1}$ are selfadjoint projections.

In view of $(2)$, to conclude that global regularity of $P_{0}$ implies global regularity of $N_{1}$ one need only know that $N_{2}$ and $N_{1}^{(t)}$ preserve global regularity. Certainly $N_{2}$ does, because the $\bar{\partial}$-Neumann operator is always regular in top dimension [16, p. 63], and we are in $\mathbf{C}^{2}$. On the other hand, by Kohn's global regularity theorem [18] for $\bar{\partial}$ there is, for each fixed positive $s$, a sufficiently large $t$ that $N_{1}^{(t)}$ preserves the Sobolev space $W^{s}(\Omega)$. Thus if for every $s$ there exists $m$ such that $P_{0}$ maps $W^{m+s}(\Omega)$ continuously into $W^{s}(\Omega)$, then $N_{1}$ has the same property. This completes the proof, since the space $C^{\infty}(\bar{\Omega})$ is the projective limit of the spaces $W^{s}(\Omega)$. (With 
a little care, using (2) and the relation $P_{0}=\chi_{0}^{(t)} P_{0}^{(t)} \chi_{0}^{(-t)}-\vartheta_{1} N_{1} \bar{\partial}_{0} \chi_{0}^{(t)} P_{0}^{(t)} \chi_{0}^{(-t)}$, one can show that $P_{0}$ and $N_{1}$ lose exactly the same number of derivatives in Sobolev norms, if any; cf. [19, p. 139].)

REMARK. It is not known if the Lemma is true for domains in $\mathbf{C}^{n}$ when $n>2$.

EXAMPLE 2. Ligocka [21, p. 201] has considered the convex Reinhardt domain $\Omega$ in $\mathbf{C}^{2}$ defined by

$$
\Omega=\left\{(z, w):|w|^{2}<\phi(|z|)\right\},
$$

where $\phi:[0,1] \rightarrow[0,1]$ is a smooth concave function such that $\phi(x)=1$ for $x$ in a neighborhood of 0 and $\phi(x)=1-x^{2}$ for $x$ in a neighborhood of 1 . The boundary contains the Cartesian product of the complex disc $\{|z|<\varepsilon\}$ with the circle $\{|w|=1\}$. This domain is globally regular for the $\bar{\partial}$-Neumann problem in view of the Lemma, because all smooth bounded pseudoconvex Reinhardt domains have globally regular Bergman projection $[\mathbf{2}, \mathbf{2 6}]$. However by Ligocka's result $[\mathbf{2 1}]$ the $\bar{\partial}$-Neumann operator for this domain is not compact in the $W^{s}(\Omega)$ topology for any $s \geq 0$.

EXAMPLE 3. Let $\Omega$ be a smooth bounded pseudoconvex domain in $\mathbf{C}^{2}$ contained in the half-space $\{(z, w): \operatorname{Re} w<0\}$, and suppose the boundary $b \Omega$ intersects the hyperplane $\{\operatorname{Re} w=0\}$ in a set $F$ with nonempty interior and is strongly pseudoconvex (finite type will do) off this plane. For instance slice the top off a strongly convex egg with a hyperplane, and round the edges. That $\Omega$ has globally regular Bergman projection $P$ follows by localizing Barrett's argument $[\mathbf{1}$, proof of Theorem 2].

For the reader's convenience I indicate the necessary modifications of his proof, which in fact shows that $P$ is bounded from the Sobolev space $W^{k}(\Omega)$ to itself for each positive integer $k$. (See [9] for a related localization argument.) We assume by induction that $\|P f\|_{k-1} \leq C\|f\|_{k-1}$, and we start with the estimate $\|P f\|_{k} \leq C\left(\left\|X^{k} P f\right\|+\|P f\|\right)$, where $X$ is any smooth vector field of type $(1,0)$ that is everywhere transverse to the boundary of $\Omega$. (Transverse derivatives control Sobolev norms of holomorphic and harmonic functions quite generally [12].) Let $\phi$ be a smooth nonnegative cut-off function that is identically equal to one in a neighborhood of $F$. By taking this neighborhood sufficiently small, we may assume that on the support of $\phi$ the field $X$ coincides with $\partial / \partial w$ and the argument of the complex inner product of $X$ with the normal to $b \Omega$ has magnitude less than say $\pi / 5 k$. In this case there exists a smooth function $a$ such that $\operatorname{Re} a^{k} \geq c>0$ and such that $(a X-\bar{X})$ is tangential to $b \Omega$ on the support of $\phi$.

Since $\bar{X}$ annihilates holomorphic functions, $(a X-\bar{X})^{k} P f$ differs from $a^{k} X^{k} P f$ by derivatives of $P f$ of order at most $k-1$. Since $a X-\bar{X}$ is tangential to the boundary on the support of $\phi$, we may integrate by parts with no boundary terms to deduce

$$
\begin{aligned}
\left\|\phi X^{k} P f\right\|^{2} & \leq C \operatorname{Re}\left\langle\phi a^{k} X^{k} P f, \phi X^{k} P f\right\rangle \\
& \leq C\left|\left\langle\phi(a X-\bar{X})^{k} P f, \phi X^{k} P f\right\rangle\right|+O\left(\|P f\|_{k-1}\|P f\|_{k}\right) \\
& \leq C\left|\left\langle P f, \phi^{2} X^{2 k} P f\right\rangle\right|+\mathcal{E} .
\end{aligned}
$$

The error terms making up $\mathcal{E}$ come from commuting derivatives and have the form $\left|\left\langle D^{j} P f, D^{l} P f\right\rangle\right|$ with $j+l \leq 2 k-1$. By the Cauchy-Riemann equations, arbitrary derivatives of holomorphic functions are effectively tangential, so we may integrate 
by parts again to conclude that the error term $\mathcal{E}$ is $O\left(\|P f\|_{k-1}\|P f\|_{k}\right)$. Recalling that $X$ agrees with $(\partial / \partial w)$ on the support of $\phi$, we obtain

$$
\begin{aligned}
\|P f\|_{k}^{2} \leq C\left(\left|\left\langle f,(\partial / \partial w)^{2 k} P f\right\rangle\right|+\mid\langle P f\right. & \left.,\left(1-\phi^{2}\right)(\partial / \partial w)^{2 k} P f\right\rangle \mid \\
& \left.+\|P f\|_{k-1}\|P f\|_{k}+\left\|(1-\phi) X^{k} P f\right\|^{2}\right) .
\end{aligned}
$$

Using integration by parts, the local regularity of $P$ at points of the support of $(1-$ $\phi)$ (these points being strongly pseudoconvex), the induction hypothesis, and the Cauchy-Schwarz inequality, we conclude

$$
\|P f\|_{k}^{2} \leq \frac{1}{2}\|P f\|_{k}^{2}+C\|f\|_{k}^{2} .
$$

This gives the required estimate if the left-hand side is finite.

To complete the proof, approximate $\Omega$ from inside by smooth strongly pseudoconvex domains $\Omega_{\varepsilon}$ (sublevel sets of a suitable defining function for $\Omega$ ). If $f \in W^{k}\left(\Omega_{\varepsilon}\right)$ then $P_{\varepsilon} f$ belongs to $W^{k}\left(\Omega_{\varepsilon}\right)$ a priori, and so the above argument gives $\left\|P_{\varepsilon} f\right\|_{k} \leq C\|f\|_{k}$ with $C$ independent of $\varepsilon$. It follows from Ramadanov's convergence theorem for the Bergman kernel function [23] that for each fixed $\delta$ the functions $P_{\varepsilon} f$ converge to $P f$ weakly in $L^{2}\left(\Omega_{\delta}\right)$. By testing against functions in $C_{0}^{\infty}\left(\Omega_{\delta}\right)$ and letting first $\varepsilon$ and then $\delta$ tend to zero one concludes that $\|P f\|_{k} \leq C\|f\|_{k}$.

Note added February 1988. The forthcoming papers [6 and 10] consider settings more general than those of Examples 1 and 2.

\section{REFERENCES}

1. David E. Barrett, Regularity of the Bergman projection and local geometry of domains, Duke Math. J. 53 (1986), 333-343.

2. Steven R. Bell and Harold P. Boas, Regularity of the Bergman projection in weakly pseudoconvex domains, Math. Ann. 257 (1981), 23-30.

3. Steve Bell and David Catlin, Boundary regularity of proper holomorphic mappings, Duke Math. J. 49 (1982), 385396.

4. Steven R. Bell and Ewa Ligocka, A simplification and extension of Fefferman's theorem on biholomorphic mappings, Invent. Math. 57 (1980), 283-289.

5. Harold P. Boas, The Szegö projection: Sobolev estimates in regular domains, Trans. Amer. Math. Soc. 300 (1987), $109-132$.

6. Harold P. Boas, So-Chin Chen, and Emil J. Straube, Exact regularity of the Bergman and Szegö projections on domains with partially transverse symmetries, preprint.

7. David W. Catlin, Global regularity of the $\bar{\partial}$-Neumann problem, Proc. Sympos. Pure Math., vol. 41, Amer. Math. Soc., Providence, R.I., 1984, pp. 39-49.

8. David Catlin, Subelliptic estimates for the $\bar{\partial}-$ Neumann problem on pseudoconvex domains, Ann. of Math. 126 (1987), 131-191.

9. So-Chin Chen, Regularity of the Bergman projection on domains with partial transverse symmetries, Math. Ann. 277 (1987), 135-140.

10. So-Chin Chen, Global regularity of the $\bar{\partial}-$ Neumann problem on circular domains, preprint.

11. John P. D'Angelo, Real hypersurfaces, orders of contact, and applications, Ann. of Math. 115 (1982), 615-637.

12. Jacqueline Detraz, Classes de Bergman de fonctions harmoniques, Bull. Soc. Math. France 109 (1981), 259-268.

13. Klas Diederich and John Erik Fornæss, Pseudoconvex domains: existence of Stein neighborhoods, Duke Math. J. 44 (1977), 641662.

14. __ Boundary regularity of proper holomorphic mappings, Invent. Math. 67 (1982), 363-384.

15. Stein neighborhoods for finite preimages of regular domain, Manuscripta Math. 50 (1985), 1127. 
16. G. B. Folland and J. J. Kohn, The Neumann problem for the Cauchy-Riemann complex, Princeton Univ. Press, Princeton, N.J., 1972.

17. J. J. Kohn, Harmonic integrals on strongly pseudo-convex manifolds. I, Ann. of Math. 78 (1963), 112-148; II, Ann. of Math. 79 (1964), 450-472.

18. $\_$, Global regularity for $\bar{\partial}$ on weakly pseudoconvex manifolds, Trans. Amer. Math. Soc. 181 (1973), 273-292.

19. __ A survey of the $\bar{\partial}-$ Neumann problem, Proc. Sympos. Pure Math., vol. 41, Amer. Math. Soc., Providence, R.I., 1984, pp. 137-145.

20. J. J. Kohn and L. Nirenberg, Non-coercive boundary value problems, Comm. Pure Appl. Math. 18 (1965), 443-492.

21. Ewa Ligocka, The regularity of weighted Bergman projections, Seminar on Deformations (Proceedings, Lodź-Warsaw 1982/84), ed. by J. Lawrynowicz, Lecture Notes in Math., vol. 1165, Springer, New York, Berlin and Heidelberg, 1985, pp. 197-203.

22. Zeev Nehari, Conformal mapping, Dover, New York, 1952.

23. I. Ramadanov, Sur une propriété de la fonction de Bergman, C. R. Acad. Bulgare Sci. (Dokl. Bolgar. Akad. Nauk) 20 (1967), 759-762.

24. R. Michael Range, The $\bar{\partial}-$ Neumann operator on the unit ball in $\mathbf{C}^{n}$, Math. Ann. 266 (1984), 449-456.

25. Nessim Sibony, Une classe de domaines pseudoconvexes, Duke Math. J. 55 (1987), 299-319.

26. Emil J. Straube, Exact regularity of Bergman, Szegó and Sobolev space projections in non pseudoconvex domains, Math. Z. 192 (1986), 117-128.

27. Matasugu Tsuji, Potential theory in modern function theory, Chelsea, New York, 1975.

Department of Mathematics, Texas A\&M University, College Station, TEXAS 77843 